\title{
LINEAR INSTABILITY IMPLIES NONLINEAR INSTABILITY FOR VARIOUS TYPES OF VISCOUS BOUNDARY LAYERS
}

\section{STABILITÉ LINÉAIRE IMPLIQUE STABILITÉ NON LINÉAIRE POUR DIVERSES COUCHES LIMITES VISQUEUSES}

\author{
B. DESJARDINS ${ }^{\mathrm{a}, *}$, E. GRENIER ${ }^{\mathrm{b}}$ \\ ${ }^{\mathrm{a}}$ C.E.A./D.I.F., BP 12, 91680 Bruyères-le-Châtel, France \\ b U.M.P.A., U.M.R. 5669, E.N.S. Lyon, 46, allée d'Italie, 69364 Lyon, France
}

Received 9 January 2001

ABSTRACT. - The aim of this paper is to give a simple proof to the fact that linear instability implies nonlinear instability for two classes of boundary layers: Ekman layers, mixed Ekman Hartmann layers. In the case of rotating fluids, we prove that linear instability of Ekman boundary layers (as studied in Lilly's work [14]) implies nonlinear instability in $L^{\infty}$ norm. This result describes the onset of turbulence at high enough Reynolds numbers. Application of these techniques to MHD models is also given.

(C) 2003 L'Association Publications de l'Institut Henri Poincaré. Published by Elsevier B.V. All rights reserved

MSC: 76E07; 35P05

RÉSUMÉ. - L'objectif de cet article est de donner une preuve simple du fait que l'instabilité linéaire entraine l'instabilité non linéaire pour deux classes de couches limites : couches limites d'Ekman, couches limites mixtes Ekman Hartmann. Dans le cas de fluides tournants, on montre que l'instabilité linéaire des couches d'Ekman (étudiée par Lilly dans [14]) implique l'instabilité non linéaire en norme $L^{\infty}$. Ce résultat décrit l'apparition de la turbulence pour des Reynolds suffisament grands. Des applications de ces techniques à des modèles de MHD sont aussi données.

(C) 2003 L'Association Publications de l'Institut Henri Poincaré. Published by Elsevier B.V. All rights reserved

\footnotetext{
* Corresponding author.

E-mail address: benoit.desjardins@mines.org (B. Desjardins).
} 


\section{Introduction}

Boundary layers appear in various physical contexts, such as the theory of rotating fluids (Ekman layers), incompressible MHD (mixed Ekman Hartmann layers), in the inviscid limit of multidimensional parabolic systems, or in the inviscid limit of NavierStokes equations near a boundary. The relevant parameter is the dimensionless Reynolds number

$$
R e=\frac{U l}{v},
$$

where $U$ denotes the typical size of the velocity outside the layer, $l$ the size of the layer and $v$ the viscosity. In classical situations, the boundary layer is expected to be stable as long as the Reynolds number remains below some critical value $R e_{c}$. Above $R e_{c}$, instabilities may appear.

From a mathematical point of view, linear and nonlinear stability of the layer can be proven when $R e<R e_{e}$, where $R e_{e}<R e_{c}$ is a critical Reynolds number associated with energy methods. Such approaches have been developped recently in a PDE's spirit, for instance in [2,7] and references therein. The problem is that in most of the applications, $R e_{e}<R e_{c}$, with an important gap between the two values. Filling the gap is the purpose of a forthcoming work. In this paper, we intend to prove that linear instability implies nonlinear instability in $L^{\infty}$ norm, namely if somewhere in the layer the Reynolds number is greater than $\operatorname{Re}_{c}$, the layer is nonlinearly unstable. We will give a general theorem and apply it to Ekman layers and Ekman Hartmann layers. We think it can be extended in a straightforward manner to multidimensional parabolic systems. The method is an improvement of the approach of [7] where instability results are proven for the incompressible Euler equations.

\section{A general instability theorem}

\subsection{Preliminaries}

We study systems of the form

$$
\partial_{t} u+Q(u, u)+\frac{L u}{\varepsilon}-\varepsilon \Delta u=0,
$$

where $u$ is vector valued, and where $Q$ if of the form

$$
Q\left(v_{1}, v_{2}\right)=\left(v_{1} \cdot \nabla\right) v_{2} \quad \text { or } \quad Q\left(v_{1}, v_{2}\right)=P\left[\left(v_{1} \cdot \nabla\right) v_{2}\right],
$$

where $P$ is the Leray projector on divergence free vector fields. Moreover, $L$ denotes a linear operator of order 0 with constant coefficients, and $\varepsilon>0$ the viscosity. Note that the case of general functions $Q$ is a straightforward adaptation of this quadratic case which is the only one detailed here for the sake of simplicity. Rotating Navier-Stokes equations, some MHD models and parabolic systems (for which $L=0$ ) enter this framework. We will consider space domains $\Omega$ of the form $\mathbb{T}^{2} \times[0,1]$ (which correspond to periodic boundary conditions in horizontal variables) or $\mathbb{R}^{2} \times[0,1]$. 
Let us first precise what we mean by a sequence of approximate solutions. We will only consider boundary layers of size $\varepsilon$.

DEFINITION 2.1. - A sequence $u^{a p p}$ (depending on $\varepsilon$ ) of functions of the form

$$
\begin{aligned}
u^{a p p}(t, x, y, z)= & \sum_{j=0}^{N} \varepsilon^{j} u_{j}^{i n t}(t, x, y)+\sum_{j=0}^{N} \varepsilon^{j} u_{j}^{B L}\left(t, x, y, \frac{z}{\varepsilon}\right) \\
& +\sum_{j=0}^{N} \varepsilon^{j} u_{j}^{B L, t}\left(t, x, y, \frac{1-z}{\varepsilon}\right)
\end{aligned}
$$

is said to be a sequence of approximate solutions of order $N$ with regularity $s$ on $[0, T]$ iffor every $0 \leqslant j \leqslant N, u_{j}^{\text {int }} \in L^{\infty}\left(0, T ; H^{s}\left(\mathbb{T}^{2}\right)\right)$, if $u_{j}^{B L}, u_{j}^{B L, t} \in L^{\infty}\left(0, T ; H^{s}\left(\mathbb{T}^{2} \times \mathbb{R}_{+}\right)\right)$ are rapidly decreasing in the last variable, and if moreover

$$
R^{a p p}=\partial_{t} u^{a p p}+Q\left(u^{a p p}, u^{a p p}\right)+\frac{L u^{a p p}}{\varepsilon}-\varepsilon \Delta u^{a p p},
$$

satisfies

$$
\left\|R^{a p p}\right\|_{L^{\infty}\left(0, T ; L^{2}(\Omega)\right)} \leqslant C_{T} \varepsilon^{N-1 / 2}
$$

for some constant $C_{T}$ independent of $\varepsilon$.

DEFINITION 2.2. - We shall say that $u^{a p p}$ is a nonlinearly unstable sequence of approximate solutions of (1) if for every arbitrarily large $s$ and $N$, and every arbitrarily small $\varepsilon>0$, there exist two solutions $u$ and $v$ of (1) with

$$
\left\|u(0, x, y)-u^{a p p}(0, x, y, z)\right\|_{H^{s}}+\left\|v(0, x, y)-u^{a p p}(0, x, y, z)\right\|_{H^{s}} \leqslant C_{s, N} \varepsilon^{N}
$$

and

$$
\begin{gathered}
\left\|u\left(T^{\varepsilon}, x, y, z\right)-v\left(T^{\varepsilon}, x, y, z\right)\right\|_{L^{\infty}} \geqslant \delta_{s}, \\
\left\|u\left(T^{\varepsilon}, x, y, z\right)-v\left(T^{\varepsilon}, x, y, z\right)\right\|_{L^{2}} \geqslant \delta_{s} \varepsilon^{3 / 2},
\end{gathered}
$$

for some times $T^{\varepsilon}$ such that $T^{\varepsilon} \leqslant C_{s} \varepsilon \log \left(2+\varepsilon^{-1}\right), C_{s}$ and $\delta_{s}$ being independent of $\varepsilon$.

Let us denote $A$ the linear operator defined by

$$
A v=Q\left(u^{a p p}, v\right)+Q\left(v, u^{a p p}\right)+\frac{L v}{\varepsilon}-\varepsilon \Delta v .
$$

The linearization of (1) around $u^{a p p}$ then writes as

$$
\partial_{t} v+A v=0
$$

Given $\left(x_{o}, y_{o}\right) \in \mathbb{R}^{2}$, we introduce the rescaled variables $\left(t_{r}, x_{r}, y_{r}, z_{r}\right)=\left(t, x-x_{o}, y-\right.$ $\left.y_{o}, z\right) / \varepsilon$, which will be used when studying small scale phenomena associated with instabilities (rescaled variables, functions and operators will be labelled with a subscript or superscript " $r$ " when necessary). We also define 


$$
\begin{aligned}
A_{x_{o}, y_{o}}^{r} v= & Q\left(u^{i n t}\left(0, x_{o}, y_{o}\right)+u_{0}^{B L}\left(0, x_{o}, y_{o}, z_{r}\right), v\right) \\
& +Q\left(v, u^{i n t}\left(0, x_{o}, y_{o}\right)+u_{0}^{B L}\left(0, x_{o}, y_{o}, z_{r}\right)\right)+L v-\Delta v,
\end{aligned}
$$

namely we freeze the $x$ and $y$ variables in the main boundary layer part of $A$ and rescale it. By rescaling Eq. (4), we formally come up at leading order with the evolution equation

$$
\partial_{t_{r}} w+A_{x_{o}, y_{o}}^{r} w=0
$$

for which Lilly [14] investigated the linear instability problem from a numerical viewpoint. We finally introduce $v=u-u^{a p p}$, which solves

$$
\partial_{t} v+A v+Q(v, v)=-R^{a p p} .
$$

The above system will be used to analyze the nonlinear evolution of perturbations $v$.

\subsection{Assumptions}

Given $k \in \mathbb{R}^{2}$, we first define the spaces $V_{k}$ by

$$
V_{k}=\left\{\exp \left(i k \cdot\left(x_{r}, y_{r}\right)\right) v\left(k, z_{r}\right), v \in C^{\infty}, i k \cdot\left(v_{1}, v_{2}\right)+\partial_{z} v_{3}=0\right\},
$$

together with a family of norms $\|v\|_{H^{\ell}}$. For simplicity of notations, we will say abusively $v \in V_{k}$ instead of $\exp \left(i k \cdot\left(x_{r}, y_{r}\right)\right) v\left(k, z_{r}\right) \in V_{k}$. Let $\Omega_{\alpha_{o}, \beta_{o}, \gamma_{o}}$ be the open set defined by

$$
\Omega_{\alpha_{o}, \beta_{o}, \gamma_{o}}=\left\{\lambda / \mathcal{R} e \lambda>\alpha_{o}\right\} \cup\left\{\lambda / \mathcal{R} e \lambda>\gamma_{o}-\beta_{o}|\mathcal{I} m \lambda|\right\}
$$

where $\alpha_{o}, \beta_{o}$ and $\gamma_{o}$ are three real positive numbers. We will assume that there exists $\left(x_{o}, y_{o}\right) \in \mathbb{R}^{2}$ such that the following assumptions hold true:

(A1) Energy estimate on the nonlinear problem:

There exists a constant $\Gamma_{0} \in \mathbb{R}_{+}$such that for all arbitrary divergence free smooth functions $u^{\prime}$, solutions $t \mapsto v(t)$ of

$$
\partial_{t} v+Q\left(u^{\prime}, v\right)+Q\left(v, u^{\prime}\right)+Q(v, v)+\frac{L v}{\varepsilon}-\varepsilon \Delta v=R,
$$

satisfy the a priori energy estimate

$$
\frac{\mathrm{d}}{\mathrm{d} t}\|v(t)\|_{L^{2}}^{2} \leqslant \Gamma_{0}\left(1+\left\|\nabla u^{\prime}\right\|_{L^{\infty}}\right)\|v(t)\|_{L^{2}}^{2}+2\|v(t)\|_{L^{2}}\|R(t)\|_{L^{2}}
$$

(A2) Linear instability:

There exists $k_{0} \in \mathbb{R}^{2}, \delta>0$, and a smooth complex valued function $k \mapsto \lambda_{1}(k)$ with positive real part for $k \in \mathcal{B}_{\delta}:=B\left(k_{0}, \delta\right) \cup B\left(-k_{0}, \delta\right)$, with $\lambda_{1}(-k)=\bar{\lambda}_{1}(k)$, and functions $v_{1}(k, \cdot)$, of positive $L^{2}$ norms for $k \in \mathcal{B}_{\delta}$, with $v_{1}(k, \cdot)=\bar{v}_{1}(k, \cdot)$ such that

$$
\left(t_{r}, x_{r}, y_{r}, z_{r}\right) \rightarrow v_{1}\left(k, z_{r}\right) \exp \left(\lambda_{1}(k) t_{r}\right) \exp \left(i k \cdot\left(x_{r}, y_{r}\right)\right)
$$


is a solution of (5) for $k \in \mathcal{B}_{\delta}$. Moreover, $\mathcal{R} e \lambda_{1}(k)$ has a maximum over $\mathcal{B}_{\delta}$ in $k_{0}$, which is nondegenerate $\left(\operatorname{Hess}\left(\mathcal{R} e \lambda_{1}\left(k_{0}\right)\right) \ll 0\right)$. We also require $\left(k, z_{r}\right) \rightarrow$ $v_{1}\left(k, z_{r}\right)$ to be smooth (for $k \in \mathcal{B}_{\delta}$ ), and define

$$
\sigma=\max _{k \in \mathcal{B}_{\delta}} \mathcal{R} e \lambda_{1}(k)
$$

(A3) If $u \in V_{k}$ and $u^{\prime} \in V_{k^{\prime}}$, then $Q\left(u, u^{\prime}\right)$ can be written under the form $u_{++}+u_{+-}+$ $u_{-+}+u_{--}$, where $u_{ \pm \pm} \in V_{ \pm k \pm k^{\prime}}$. In addition,

$$
\left\|u_{ \pm \pm}\right\|_{\ell-\sigma_{o}} \leqslant C_{k, k^{\prime}, \ell}\|u\|_{\ell}\left\|u^{\prime}\right\|_{\ell},
$$

where $\sigma_{o}$ is independent of $k, k^{\prime}$ and $\ell, C_{k, k^{\prime}, \ell}$ being locally bounded in $k$ and $k^{\prime}$.

(A4) Estimate on the resolvent of $A_{x_{o}, y_{o}}^{r}$ :

Let

$$
R(\lambda, k)=\left(\lambda+A_{x_{o}, y_{o}}^{r}\right)^{-1}
$$

We assume that for any $\alpha_{o}>\sigma$ there exist $\beta_{o}$ and $\gamma_{o}$ such that $R$ is well defined on $\bar{\Omega}_{\alpha_{o}, \beta_{o}, \gamma_{o}}$ and satisfies: for every arbitrarily large $\alpha$, every arbitrarily large $s$, and every $\lambda \in \bar{\Omega}_{\alpha_{o}, \beta_{o}, \gamma_{o}}$,

$$
\left\|\partial_{k}^{\alpha} R(\lambda, k)\right\|_{H^{s} \rightarrow H^{s}} \leqslant C_{R, \alpha}(\lambda, k)
$$

where $C_{R, \alpha}$ has at most a polynomial growth in $k$ and is bounded uniformly on sets of the form $\mathcal{R} e \lambda<\lambda^{\prime}$ for all arbitrarily large $\lambda^{\prime}>\alpha_{o}$. We allow $\beta_{o}$ and $\gamma_{o}$ to depend on $k$, in a locally bounded way.

THEOREM 2.3. - Under assumptions (A1), (A2), (A3) and (A4), the sequence $u^{a p p}$ of approximate solutions is nonlinearly unstable.

Remarks. - (A1) is a very rough $L^{2}$ estimate on the nonlinear system. Let us emphasize that $\Gamma_{0}$ can be large, and actually much larger than $\sigma$. (A2) is the linear instability and stems from physical and numerical analyses. Assumption (A3) is usually straightforward and applies to nonlinearities $Q$ of the form $Q\left(v_{1}, v_{2}\right)=\left(v_{1} \cdot \nabla\right) v_{2}$. Assumption (A4) is the difficult point to check. However, in applications it often reduces to a simple verification on a system of ordinary differential equations. Note that this assumption is completely different from the approach of [7].

Note also that the proof of Theorem 2.3 provides in fact much more information than the theorem itself. In particular, it gives a precise description of the solution until the instability time $T_{r}^{\varepsilon}$ : for $t_{r}$ near $T_{r}^{\varepsilon}$, the solution mainly behaves like

$$
u \sim u_{r}^{a p p}+\frac{4 \pi}{t_{r}} \mathcal{R} e\left(v_{1}\left(k_{0}, z_{r}\right) \exp \left(i k_{0} \cdot\left(x_{r}, y_{r}\right)\right) \exp \left(\lambda_{1}\left(k_{0}\right) t_{r}\right)\right) \text {, }
$$

for $\left|\left(x_{r}, y_{r}\right)+\mathcal{I} m \partial_{k} \lambda_{1}\left(k_{0}\right) t_{r}\right| \ll \sqrt{t_{r}}$. Therefore, near $T_{r}^{\varepsilon}$, only the most unstable modes $\pm k_{0}$ emerge, after a travel at speed $-\mathcal{I} m \partial_{k} \lambda_{1}\left(k_{0}\right)$. Physically speaking, if one perturbes $u^{a p p}$ by a very small noise, waves of wavenumbers $k_{0}$ grow more rapidly than the other waves, and travel to create the instability. In general, $\mathcal{I} m \partial_{k} \lambda_{1}\left(k_{0}\right) \neq 0$ which leads to a so called "convective instability". 


\subsection{Proof}

\subsubsection{Construction of an unstable wavepacket}

The first step is to construct an unstable wavepacket which is localized in space and exponentially increasing in time, and to derive $L^{2}$ and $L^{\infty}$ estimates on it. Let us first observe that the horizontal invariance allows to restrict to the case $x_{o}=y_{o}=0$. Let $\eta>0$ small enough such that $\eta<\delta$. Let $\mathcal{B}_{\eta}:=B\left(k_{0}, \eta\right) \cup B\left(-k_{0}, \eta\right)$, and $\phi$ be a smooth real valued function supported in $\mathcal{B}_{\eta}$, with $\phi\left(k_{0}\right)=1$ and $\phi(-k)=\bar{\phi}(k)$. We take

$$
u_{0}\left(t_{r}, x_{r}, y_{r}, z_{r}\right)=\int_{\mathcal{B}_{\eta}} \phi(k) v_{1}\left(k, z_{r}\right) \exp \left(\lambda_{1}(k) t_{r}\right) \exp \left(i k \cdot\left(x_{r}, y_{r}\right)\right) \mathrm{d} k,
$$

which is a real valued solution of (5) in view of assumption (A2). Let us also remark that

$$
\lambda_{1}(k)=\lambda_{1}\left(k_{0}\right)+\nabla_{k} \lambda_{1}\left(k_{0}\right) \cdot\left(k-k_{0}\right)-\left|k-k_{0}\right|^{2} \frac{\alpha\left(k-k_{0}\right)}{2},
$$

where $0<\underline{\alpha} \leqslant \operatorname{Re} \alpha(\cdot) \leqslant \bar{\alpha}$ in $B(0, \eta)$. Moreover, denoting

$$
\left(\tilde{x}_{r}, \tilde{y}_{r}\right)=\frac{\left(x_{r}, y_{r}\right)-i t_{r} \nabla_{k} \lambda_{1}\left(k_{0}\right)}{\sqrt{t_{r}}},
$$

we rewrite $u_{0}$ as follows

$$
\begin{aligned}
u_{0}\left(t_{r}, x_{r}, y_{r}, z_{r}\right)= & 2 \mathcal{R} e\left\{\exp \left(\lambda_{1}\left(k_{0}\right) t_{r}+i k_{0} \cdot\left(x_{r}, y_{r}\right)\right) \int_{B\left(k_{0}, \eta\right)} \phi(k) v_{1}\left(k, z_{r}\right)\right. \\
& \left.\times \exp \left(i\left(k-k_{0}\right) \sqrt{t_{r}} \cdot\left(\tilde{x}_{r}, \tilde{y}_{r}\right)-\left|k-k_{0}\right|^{2} t_{r} \frac{\alpha\left(k-k_{0}\right)}{2}\right) \mathrm{d} k\right\} \\
= & \frac{2}{t_{r}} \mathcal{R} e\left\{\exp \left(\lambda_{1}\left(k_{0}\right) t_{r}+i k_{0} \cdot\left(x_{r}, y_{r}\right)\right)\right. \\
& \times \int_{B\left(0, \eta \sqrt{t_{r}}\right)} \phi\left(k_{0}+\frac{\kappa}{\sqrt{t_{r}}}\right) v_{1}\left(k_{0}+\frac{\kappa}{\sqrt{t_{r}}}, z_{r}\right) \\
& \left.\times \exp \left(i \kappa \cdot\left(\tilde{x}_{r}, \tilde{y}_{r}\right)-\frac{|\kappa|^{2}}{2} \alpha\left(\frac{\kappa}{\sqrt{t_{r}}}\right)\right) \mathrm{d} \kappa\right\} .
\end{aligned}
$$

Classical arguments yield the following convergence uniformly in $z_{r} \in\left[0, Z_{0}\right]$ and $\left|\left(\tilde{x}_{r}, \tilde{y}_{r}\right)\right| \leqslant A_{0}$

$$
\begin{aligned}
& \int_{B\left(0, \eta \sqrt{t_{r}}\right)} \phi\left(k_{0}+\frac{\kappa}{\sqrt{t_{r}}}\right) v_{1}\left(k_{0}+\frac{\kappa}{\sqrt{t_{r}}}, z_{r}\right) \exp \left(i \kappa \cdot\left(\tilde{x}_{r}, \tilde{y}_{r}\right)\right. \\
& \left.-\frac{|\kappa|^{2}}{2} \alpha\left(\frac{\kappa}{\sqrt{t_{r}}}\right)+\frac{\left|\left(\tilde{x}_{r}, \tilde{y}_{r}\right)\right|^{2}}{2 \alpha(0)}\right) \mathrm{d} \kappa \rightarrow v_{1}\left(k_{0}, z_{r}\right) \frac{2 \pi}{\alpha(0)} \quad \text { when } t_{r} \rightarrow \infty .
\end{aligned}
$$


As a result,

$$
u_{0}\left(t_{r}, x_{r}, y_{r}, z_{r}\right) \sim \frac{4 \pi}{t_{r}} \mathcal{R} e\left\{\frac{v_{1}\left(k_{0}, z_{r}\right)}{\alpha(0)} \exp \left(-\frac{\left|\left(\tilde{x}_{r}, \tilde{y}_{r}\right)\right|^{2}}{2 \alpha(0)}+\lambda_{1}\left(k_{0}\right) t_{r}+i k_{0} \cdot\left(x_{r}, y_{r}\right)\right)\right\}
$$

uniformly in $z_{r} \in\left[0, Z_{0}\right]$ and $\left|\left(\tilde{x}_{r}, \tilde{y}_{r}\right)\right| \leqslant A_{0}$. Denoting $\varphi\left(z_{r}\right)=\operatorname{Arg}\left(v_{1}\left(k_{0}, z_{r}\right) / \alpha(0)\right)$, $1 / \alpha(0)=\alpha_{1}+i \alpha_{2}, \omega_{0}=\operatorname{I} m \lambda_{1}\left(k_{0}\right)$, we deduce that

$$
\begin{aligned}
u_{0}\left(t_{r}, x_{r}, y_{r}, z_{r}\right) \sim & \frac{4 \pi}{t_{r}} \exp \left(-\alpha_{1} \frac{\left|\left(\tilde{x}_{r}, \tilde{y}_{r}\right)\right|^{2}}{2}+\mathcal{R} e \lambda_{1}\left(k_{0}\right) t_{r}\right) \frac{\left|v_{1}\left(k_{0}, z_{r}\right)\right|}{|\alpha(0)|} \\
& \times \cos \left(\omega_{0} t_{r}+k_{0} \cdot\left(x_{r}, y_{r}\right)-\alpha_{2} \frac{\left|\left(\tilde{x}_{r}, \tilde{y}_{r}\right)\right|^{2}}{2}+\varphi\left(k_{0}, z_{r}\right)\right) .
\end{aligned}
$$

As a consequence, we have for $t_{r}$ large enough

$$
\left|u_{0}\left(t_{r}, x_{r}, y_{r}, z_{r}\right)\right| \leqslant \frac{C}{t_{r}} \exp \left(\mathcal{R} e \lambda_{1}\left(k_{0}\right) t_{r}\right)
$$

uniformly in $z_{r} \in\left[0, Z_{0}\right]$ and $\left|\left(\tilde{x}_{r}, \tilde{y}_{r}\right)\right| \leqslant A_{0}$, and

$$
\left\|u_{0}\left(t_{r}\right)\right\|_{L^{2}\left(\left|\left(\tilde{x}_{r}, \tilde{y}_{r}\right)\right| \leqslant A_{0}, z_{r} \in\left[0, Z_{0}\right]\right)} \geqslant \frac{C_{o}}{\sqrt{t_{r}}} \exp \left(\mathcal{R} e \lambda_{1}\left(k_{0}\right) t_{r}\right)
$$

Moreover, we have

$$
\left\|u_{0}\left(t_{r}\right)\right\|_{L^{2}}^{2}=C \int_{\mathcal{B}_{\eta}}|\phi(k)|^{2}\left\|v_{1}(k, .)\right\|_{L^{2}}^{2} \exp \left(2 \mathcal{R} e \lambda_{1}(k) t_{r}\right) \mathrm{d} k
$$

Since, for $\eta$ small enough, $\lambda_{1}$ has a unique nondegenerate maximum in $B\left(k_{0}, \eta\right)$ at $k=k_{0}$, easy arguments yield

$$
\begin{aligned}
\left\|u_{0}\left(t_{r}\right)\right\|_{L^{2}} & \sim \frac{C_{1}}{\sqrt{t_{r}}} \exp \left(\mathcal{R} e \lambda_{1}\left(k_{0}\right) t_{r}\right), \\
\left\|u_{0}\left(t_{r}\right)\right\|_{L^{\infty}} & \leqslant \frac{C_{1}^{\prime}}{t_{r}} \exp \left(\mathcal{R} e \lambda_{1}\left(k_{0}\right) t_{r}\right),
\end{aligned}
$$

as $t_{r} \rightarrow+\infty$.

\subsubsection{Construction of an approximate solution}

We will construct a new family of approximate solutions $u^{\varepsilon}$ of the form

$$
u^{\varepsilon}=u_{r}^{a p p}+\varepsilon^{N} \sum_{j=0}^{M} \varepsilon^{j} u_{j}
$$

where each $u_{j}$ is a sum of functions $\left(u_{j, \ell, \ell^{\prime}}\right)_{1 \leqslant \ell, \ell^{\prime} \leqslant j+1}$

$$
u_{j}=\sum_{\ell, \ell^{\prime}} u_{j, \ell, \ell^{\prime}}
$$


Here, $u_{j, \ell, \ell^{\prime}}$ depends on $\varepsilon$ (the dependence is omitted in the notation) and can be expressed as follows

$$
\begin{aligned}
u_{j, \ell, \ell^{\prime}}= & \int_{\left(k_{1}, \ldots, k_{\ell}\right) \in \mathcal{B}_{\eta}^{\ell},\left(\tilde{k}_{1}, \ldots, \tilde{k}_{\ell^{\prime}}\right) \in B(0, \eta)^{\ell^{\prime}}} u_{j, k_{1}, \ldots, k_{\ell}, \tilde{k}_{1}, \ldots, \tilde{k}_{\ell^{\prime}}}\left(t_{r}, z_{r}\right) \\
& \times \exp \left(i\left(k_{1}+\cdots+k_{\ell}+\tilde{k}_{1}+\cdots+\tilde{k}_{\ell^{\prime}}\right)\left(x_{r}, y_{r}\right)\right) \mathrm{d} k_{1} \ldots \mathrm{d} k_{\ell} \mathrm{d} \tilde{k}_{1} \ldots \mathrm{d} \tilde{k}_{\ell^{\prime}} .
\end{aligned}
$$

We will show by induction the following estimate

$$
\left\|u_{j, k_{1}, \ldots, k_{\ell}, \tilde{k}_{1}, \ldots, \tilde{k}_{\ell^{\prime}}}\left(t_{r}\right)\right\|_{s} \leqslant C_{j, s} \exp \left(\mathcal{R} e\left(\lambda_{1}\left(k_{1}\right)+\cdots+\lambda_{1}\left(k_{\ell}\right)+\ell^{\prime} \bar{\delta}\right) t_{r}\right)
$$

for every $s \geqslant 0$, where $\bar{\delta}$ is small enough. Moreover, all the non zero $u_{j, \ell, \ell^{\prime}}$ will satisfy

$$
\ell N+\ell^{\prime}=N+j, \quad \text { and } \quad \ell \geqslant 1 .
$$

We will finally show that $u$ is smooth in $\left(k_{1}, \ldots, k_{\ell}\right)$ in the following sense: for every multiindex $\alpha$ of length $\ell$, for every multiindex $\alpha^{\prime}$ of length $\ell^{\prime}$ and every $s$,

$$
\begin{aligned}
& \left\|\partial_{k}^{\alpha} \partial_{\tilde{k}}^{\alpha^{\prime}} u_{j, k_{1}, \ldots, k_{\ell}, \tilde{k}_{1}, \ldots, \tilde{k}_{\ell^{\prime}}}\left(t_{r}\right)\right\|_{s} \\
& \quad \leqslant C_{j, s, \alpha, \alpha^{\prime}} \exp \left(\operatorname{Re} e\left(\lambda_{1}\left(k_{1}\right)+\cdots+\lambda_{1}\left(k_{\ell}\right)+\ell^{\prime} \bar{\delta}\right) t_{r}\right) .
\end{aligned}
$$

This smoothness will guarantee the fast decrease of $u_{j}$ in the tangential variables $\left(x_{r}, y_{r}\right)$. The functions $u_{j, \ell, \ell^{\prime}}$ are constructed by induction on $j$ in the next subsection. Roughly speaking, $\ell^{\prime}$ controls the interactions with $u_{r}^{a p p}$, and $\ell$ takes account of the quadratic interactions of $u^{\varepsilon}-u_{r}^{a p p}$.

Let us for the moment derive from (20) some $L^{\infty}$ and $L^{2}$ estimates on $u_{j}$. First, we have

$$
\begin{aligned}
\left\|u_{j, \ell, \ell^{\prime}}\left(t_{r}\right)\right\|_{L^{\infty}} & \leqslant C \exp \left(\ell^{\prime} \bar{\delta} t_{r}\right)\left(\int_{\mathcal{B}_{\eta}} \exp \left(\mathcal{R} e \lambda_{1}(k) t_{r}\right) \mathrm{d} k\right)^{\ell} \\
& \leqslant \frac{C}{t_{r}^{\ell}} \exp \left(\ell^{\prime} \bar{\delta} t_{r}\right) \exp \left(\mathcal{R} e \lambda_{1}\left(k_{0}\right) \ell t_{r}\right) .
\end{aligned}
$$

Let us observe that for $\bar{\delta}$ small enough and $t_{r}$ bounded away from zero, we have

$$
\exp \left(\ell^{\prime} \bar{\delta} t_{r}\right) \leqslant \frac{C}{t_{r}^{\ell^{\prime} / N}} \exp \left(\mathcal{R} e \lambda_{1}\left(k_{0}\right) \frac{\ell^{\prime}}{N} t_{r}\right)
$$

Therefore,

$$
\left\|u_{j, \ell, \ell^{\prime}}\left(t_{r}\right)\right\|_{L^{\infty}} \leqslant \frac{C}{t_{r}^{1+j / N}} \exp \left(\mathcal{R} e \lambda_{1}\left(k_{0}\right)\left(1+\frac{j}{N}\right) t_{r}\right)
$$

Moreover,

$$
\left\|\int_{k_{1}+\cdots+k_{\ell}+\tilde{k}_{1}+\cdots+\tilde{k}_{\ell^{\prime}}=\ell k} u_{j, k_{1}, \ldots, k_{\ell}, \tilde{k}_{1}, \ldots, \tilde{k}_{\ell^{\prime}}}\left(t_{r}\right)\right\|_{L^{2}}
$$




$$
\leqslant C \int_{k_{1}+\cdots+k_{\ell}+\tilde{k}_{1}+\cdots+\tilde{k}_{\ell^{\prime}=\ell k}} \exp \left(\bar{\delta} \ell^{\prime} t_{r}\right) \exp \left(\mathcal{R} e \sum_{i=1}^{\ell} \lambda_{1}\left(k_{i}\right) t_{r}\right) .
$$

If $\ell^{\prime}=0$, the left hand side of (25) is bounded by

$$
\begin{aligned}
& \leqslant C \int_{k_{1}+\cdots+k_{\ell}=\ell k} \exp \left(\left(\mathcal{R} e\left(\ell \lambda_{1}\left(k_{0}\right)\right)-\beta \ell\left(k_{0}-k\right)^{2}-\beta \sum_{i=1}^{\ell}\left(k-k_{i}\right)^{2}\right) t_{r}\right) \\
& \leqslant \frac{C_{\ell}}{t_{r}^{\ell-1}} \exp \left(\left(\ell \mathcal{R} e \lambda_{1}\left(k_{0}\right)-\beta \ell\left(k_{0}-k\right)^{2}\right) t_{r}\right)
\end{aligned}
$$

for some $\beta>0$. Hence,

$$
\left\|u_{j, \ell, 0}\left(t_{r}\right)\right\|_{L^{2}} \leqslant \frac{C_{\ell}}{t_{r}{ }^{\ell}} \sqrt{t_{r}} \exp \left(\mathcal{R} e \lambda_{1}\left(k_{0}\right) \ell t_{r}\right) .
$$

If $\ell^{\prime} \neq 0$, we use a more crude estimate

$$
\left\|u_{j, \ell, \ell^{\prime}}\left(t_{r}\right)\right\|_{L^{2}} \leqslant C \exp \left(\bar{\delta} \ell^{\prime} t_{r}\right) \exp \left(\mathcal{R} e \lambda_{1}\left(k_{0}\right) \ell t_{r}\right)
$$

so that recalling (23), we obtain

$$
\left\|u_{j, \ell, \ell^{\prime}}\left(t_{r}\right)\right\|_{L^{2}} \leqslant \frac{C_{\ell}}{t_{r}{ }^{1+j / N}} \sqrt{t_{r}} \exp \left(\mathcal{R} e \lambda_{1}\left(k_{0}\right)\left(1+\frac{j}{N}\right) t_{r}\right)
$$

estimate which is true in the case $\ell^{\prime}=0$ as well.

\subsubsection{Error terms}

Let us now detail the construction of the new approximate solution. First denoting

$$
w=\sum_{j=0}^{M} \varepsilon^{j} u_{j}
$$

we obtain

$$
\partial_{t_{r}} w+A_{x_{o}, y_{o}}^{r} w+\varepsilon^{N} Q(w, w)+\left(A^{r}-A_{x_{o}, y_{o}}^{r}\right) w+\frac{R^{a p p}}{\varepsilon^{N}}=0,
$$

so that expanding the difference $\left(A^{r}-A_{x_{o}, y_{o}}^{r}\right) w$ in Taylor series yields in the particular case when $Q\left(v_{1}, v_{2}\right)=P\left(v_{1} \cdot \nabla v_{2}\right)$

$$
\begin{aligned}
& \partial_{t_{r}} u_{j}+A_{x_{o}, y_{o}}^{r} u_{j}+\sum_{j^{\prime}+j^{\prime \prime}+N=j} Q\left(u_{j^{\prime}}, u_{j^{\prime \prime}}\right) \\
& \quad+\mathcal{F}_{x_{r}, y_{r}}^{-1} P\left\{\sum _ { j ^ { \prime } + j ^ { \prime \prime } = j } \sum _ { \alpha = ( \alpha _ { o } , \alpha _ { 1 } ) , | \alpha | = j ^ { \prime } } \left\{(-i)^{|\alpha|} \frac{\partial^{\alpha} \nabla u^{a p p}}{\alpha !}\left(0,0,0, z_{r}\right) t^{\alpha_{o}} \partial_{k}^{\alpha_{1}} u_{j^{\prime \prime}}\right.\right. \\
& \left.\left.\quad+(-i)^{|\alpha|} \frac{\partial^{\alpha} u^{a p p}}{\alpha !}\left(0,0,0, z_{r}\right) t^{\alpha_{o}} \partial_{k}^{\alpha_{1}}\left[\begin{array}{c}
i k \\
\partial_{z_{r}}
\end{array}\right] u_{j^{\prime \prime}}\right\}\right\}=0 .
\end{aligned}
$$


Thus, $\bar{u}_{j, k_{1}, \ldots, k_{\ell}, \tilde{k}_{1}, \ldots, \tilde{k}_{\ell^{\prime}}}$ will be solution of equations of the form

$$
\partial_{t_{r}} \bar{u}_{j, k_{1}, \ldots, k_{\ell}, \tilde{k}_{1}, \ldots, \tilde{k}_{\ell^{\prime}}}+A_{x_{o}, y_{o}}^{r} \bar{u}_{j, k_{1}, \ldots, k_{\ell}, \tilde{k}_{1}, \ldots, \tilde{k}_{\ell^{\prime}}}+R_{j, k_{1}, \ldots, k_{\ell}, \tilde{k}_{1}, \ldots, \tilde{k}_{\ell^{\prime}}}=0
$$

where $R_{j, k_{1}, \ldots, k_{\ell}, \tilde{k}_{1}, \ldots, \tilde{k}_{\ell^{\prime}}}$ is the sum of error terms of order $\varepsilon^{N+j}$ generated by inserting $u^{\varepsilon}$ into the rescaled version of (1). These error terms have three different origins.

The first family is of the form

$$
R^{1}=Q\left(\bar{u}_{j^{\prime}, k_{1}, \ldots, k_{\ell_{1}}, \tilde{k}_{1}, \ldots, \tilde{k}_{\ell_{1}^{\prime}}}, \bar{u}_{j^{\prime \prime}, k_{\ell_{1}+1}, \ldots, k_{\ell}, k_{\ell_{1}^{\prime}+1}, \ldots, \tilde{k}_{\ell^{\prime}}}\right)
$$

with $N+j^{\prime}+j^{\prime \prime}=j, 1 \leqslant \ell_{1} \leqslant \ell$ and $1 \leqslant \ell_{1}^{\prime} \leqslant \ell^{\prime}$, coming from quadratic interactions between $u_{j^{\prime}, \ell_{1}, \ell_{1}^{\prime}}$ and $u_{j^{\prime \prime}, \ell-\ell_{1}, \ell^{\prime}-\ell_{1}^{\prime}}$. We deduce from (20), (22) and (A3)

$$
\left\|R^{1}\left(t_{r}\right)\right\|_{s} \leqslant C_{s} \exp \left(\mathcal{R} e\left(\lambda_{1}\left(k_{1}\right)+\cdots+\lambda_{1}\left(k_{\ell}\right)+\bar{\delta} \ell^{\prime}\right) t_{r}\right)
$$

and similarly for all its derivatives with respect to the wave numbers $k_{i}$ and $\tilde{k}_{i}$. But using the resolvent we have

$$
\begin{aligned}
& \bar{u}_{j, k_{1}, \ldots, k_{\ell}, \tilde{k}_{1}, \ldots, \tilde{k}_{\ell^{\prime}}\left(t_{r}\right)} \\
& =\int_{0}^{t_{r}} \mathrm{e}^{-\left(t_{r}-\tau\right) A_{x_{0}, y_{0}}^{r} R^{1}(\tau) \mathrm{d} \tau} \\
& =-\frac{1}{2 i \pi} \int_{0}^{t_{r}} \int_{\Gamma} \mathrm{e}^{\left(t_{r}-\tau\right) \lambda} R\left(\lambda, k_{1}+\cdots+k_{\ell}+\tilde{k}_{1}+\cdots+\tilde{k}_{\ell^{\prime}}\right) R^{1}(\tau) \mathrm{d} \lambda \mathrm{d} \tau
\end{aligned}
$$

where $\Gamma=\partial \Omega$. Using assumption (A4) we get (20) and (22). Note that (21) is preserved.

The second family arises from the interactions with $A^{r}-A_{x_{o}, y_{o}}^{r}$ and is of the form

$$
R^{2}=\left(A^{r}-A_{x_{o}, y_{o}}^{r}\right) \bar{u}_{j-1, k_{1}, \ldots, k_{\ell}, \tilde{k}_{1}, \ldots, \tilde{k}_{\ell^{\prime}}} .
$$

Here there is a technical difficulty. We have to apply the Fourier transform to $R^{2}$ and to take the frequency $k_{1}+\cdots+k_{\ell}+\tilde{k}_{1}+\cdots+\tilde{k}_{\ell^{\prime}}$ where $\tilde{k}_{\ell^{\prime}} \in B(0, \eta)$, therefore we have to throw away parts of the Fourier transform of $u_{r}^{a p p}$ with $\left|\tilde{k}_{\ell^{\prime}}\right| \geqslant B(0, \eta)$. However as $u_{r}^{a p p}$ is smooth in horizontal variables, and having in mind the change of scale between $(x, y)$ and $\left(x_{r}, y_{r}\right)$ the terms omitted are $\mathrm{O}\left(\varepsilon^{\infty}\right)$ and will be forgotten. Note that again (21) is preserved.

The third family finally stems from $R^{3}=-R^{a p p} / \varepsilon^{N}$ and can be treated in a similar and actually easier way.

\subsubsection{Conclusion}

At this point, we have contructed an approximate solution $u^{\varepsilon}$ which in rescaled variables solves

$$
\partial_{t_{r}} u^{\varepsilon}+Q\left(u^{\varepsilon}, u^{\varepsilon}\right)-\Delta_{r} u^{\varepsilon}+L u^{\varepsilon}=R^{\varepsilon},
$$

where the remainder $R^{\varepsilon}$ satisfies 


$$
\begin{aligned}
& \left\|R^{\varepsilon}\left(t_{r}\right)\right\|_{L^{2}} \leqslant \frac{C \varepsilon^{N P}}{t_{r}^{P}} \sqrt{t_{r}} \exp \left(P \mathcal{R} e \lambda_{1}\left(k_{0}\right) t_{r}\right) \leqslant C \sqrt{t_{r}} \mathcal{E}^{P}\left(t_{r}\right), \\
& \text { where } P=1+\frac{M+1}{N} \text { and } \mathcal{E}\left(t_{r}\right)=\frac{\varepsilon^{N}}{t_{r}} \exp \left(\mathcal{R} e \lambda_{1}\left(k_{0}\right) t_{r}\right) .
\end{aligned}
$$

Let $T_{0}$ such that $\mathcal{E}\left(T_{0}\right)=1$. We first want to compare $u^{\varepsilon}$ with $u_{r}^{a p p}$. Let, for $A>0$,

$$
\Omega_{A}=\left\{\left|\left(x_{r}, y_{r}\right)+\mathcal{I} m \nabla_{k} \lambda_{1}\left(k_{0}\right) t_{r}\right| \leqslant A \sqrt{t_{r}},\left|z_{r}\right| \leqslant A\right\} .
$$

Using (15), there exists $A>0$ such that for every $t_{r} \geqslant 0$,

$$
\left\|u_{0}\left(t_{r}\right)\right\|_{L^{2}\left(\Omega_{A}\right)} \geqslant C_{o} \sqrt{t_{r}} \mathcal{E}\left(t_{r}\right)
$$

for some $C_{o}>0$. We deduce from (27) and (32) that

$$
\left\|\left(u^{\varepsilon}-u_{r}^{a p p}\right)\left(t_{r}\right)\right\|_{L^{2}\left(\Omega_{A}\right)} \geqslant C_{o} \sqrt{t_{r}} \mathcal{E}\left(t_{r}\right)-\sum_{j=1}^{M} C_{j} \sqrt{t_{r}} \mathcal{E}^{1+j / N}\left(t_{r}\right),
$$

for some constants $C_{j}$ having at most polynomial growth in $j$. Thus, for $t_{r} \leqslant T_{1}=$ $T_{0}-\sigma_{1}$ with $\sigma_{1}$ large enough, but independent of $\varepsilon$,

$$
\left\|\left(u^{\varepsilon}-u_{r}^{a p p}\right)\left(t_{r}\right)\right\|_{L^{2}\left(\Omega_{A}\right)} \geqslant \frac{C_{o}}{2} \sqrt{t_{r}} \mathcal{E}\left(t_{r}\right) .
$$

Let us now define $u$ as a solution of

$$
\partial_{t} u+Q(u, u)+\frac{L u}{\varepsilon}-\varepsilon \Delta u=0
$$

with initial data $u_{0}^{a p p}+\varepsilon^{N} u_{0}$. We will work in rescaled variables until the end of this section. Let $v=u-u^{\varepsilon}$. It satisfies

$$
\partial_{t_{r}} v+Q\left(u^{\varepsilon}, v\right)+Q\left(v, u^{\varepsilon}\right)+Q(v, v)+L v-\Delta_{r} v=-R^{\varepsilon}
$$

hence using (A1),

$$
\frac{\mathrm{d}}{\mathrm{d} t}\|v(t)\|_{L^{2}}^{2} \leqslant \Gamma_{0}\left(2+\left\|\nabla u^{\varepsilon}\right\|_{L^{\infty}}\right)\|v(t)\|_{L^{2}}^{2}+C\left\|R^{\varepsilon}(t)\right\|_{L^{2}}^{2}
$$

But we have in view of (23)

$$
\left\|\nabla u^{\varepsilon}\left(t_{r}\right)\right\|_{L^{\infty}} \leqslant\left\|\nabla u_{r}^{a p p}\left(t_{r}\right)\right\|_{L^{\infty}}+\sum_{j=0}^{M} C_{j}^{\prime \prime} \mathcal{E}^{1+j / N}\left(t_{r}\right)
$$

where $C_{j}^{\prime \prime}$ has at most polynomial growth in $j$. Thus, for $t_{r} \leqslant T_{2}=T_{0}-\sigma_{2}$ with $\sigma_{2}>\sigma_{1}$ large enough but independent of $\varepsilon$,

$$
\left\|\nabla u^{\varepsilon}\left(t_{r}\right)\right\|_{L^{\infty}} \leqslant 2\left\|\nabla u_{r}^{a p p}\left(t_{r}\right)\right\|_{L^{\infty}}+1
$$


Let $M$ such that

$$
2 \frac{M+1}{N} \mathcal{R} e \lambda_{1}\left(k_{0}\right)>\Gamma_{0}\left(3+2 \sup _{t_{r} \in\left(0, T_{0}\right)}\left\|\nabla u_{r}^{a p p}\left(t_{r}\right)\right\|_{L^{\infty}}\right) .
$$

For $t_{r} \leqslant T_{3}=T_{0}-\sigma_{2}$ we therefore have, after time scaling,

$$
\begin{aligned}
\frac{\mathrm{d}}{\mathrm{d} t_{r}}\left\|v\left(t_{r}\right)\right\|_{L^{2}}^{2} \leqslant & \Gamma_{0}\left(3+2 \sup _{t_{r} \in\left(0, T_{0}\right)}\left\|\nabla u_{r}^{a p p}\left(t_{r}\right)\right\|_{L^{\infty}}\right)\left\|v\left(t_{r}\right)\right\|_{L^{2}}^{2} \\
& +\frac{C \varepsilon^{2 N P} t_{r}}{t_{r}^{2 P}} \exp \left(2 P \mathcal{R} e \lambda_{1}\left(k_{0}\right) t_{r}\right) .
\end{aligned}
$$

Using the fact that $v(0)=0$ we have

$$
\left\|v\left(t_{r}\right)\right\|_{L^{2}} \leqslant C^{\prime} \sqrt{t_{r}} \mathcal{E}^{P}\left(t_{r}\right)
$$

Indeed, if $\lambda_{2}<\lambda_{3}$, a function $\phi$ satisfying $\phi(0)=0$ and

$$
\frac{\mathrm{d}}{\mathrm{d} t} \phi \leqslant \lambda_{2} \phi+\frac{\exp \left(\lambda_{3} t\right)}{1+t^{N}}
$$

verifies in view of integration by parts arguments

$$
\phi \leqslant C \exp \left(\lambda_{3} t\right) \int_{0}^{t} \frac{\exp \left(\left(\lambda_{2}-\lambda_{3}\right)(t-\tau)\right)}{1+\tau^{N}} \mathrm{~d} \tau \leqslant C \frac{\exp \left(\lambda_{3} t\right)}{1+t^{N}}
$$

Now for $T_{r}=T_{0}-\sigma_{3}$, with $\sigma_{3} \geqslant \sigma_{2}$ large enough,

$$
\left\|\left(u-u^{\varepsilon}\right)\left(T_{r}\right)\right\|_{L^{2}\left(\Omega_{A}\right)} \geqslant\left\|\left(u^{\varepsilon}-u_{r}^{a p p}\right)\left(T_{r}\right)\right\|_{L^{2}\left(\Omega_{A}\right)}-\left\|v\left(T_{r}\right)\right\|_{L^{2}} \geqslant \sigma_{o}\left(T_{r}\right)^{1 / 2}
$$

with $\sigma_{o}$ independent of $\varepsilon$. Now repeating the construction with $u_{0}=0$, we get two solutions which separate, which ends up the proof: the Lebesgue measure of $\Omega_{A}$ is of order $C T_{r}$, which allows to get the claimed $L^{\infty}$ bounds. The $L^{2}$ estimate in original variables also follows from straightforward scaling arguments.

\section{Some results of spectral theory}

To prove assumption (A4) we will mainly use two results. First $A_{x_{o}, y_{o}}^{r}$ is a compact perturbation of the Laplace operator, and hence is a sectorial operator [12]. Second we will use results of Shizuta and Vidav [18,19] that we now recall (see other applications in $[10,11])$. Let us first begin by a definition.

DEFINITION 3.1. - Let A be a linear operator which generates a strongly continuous semigroup $t \mapsto \exp (-t A)$. We say that an operator $K$ is $A$-smoothing if

(a) $\exp (-t A) K$ is compact for every $t>0$,

(b) $t \rightarrow \exp (-t A) K$ is continuous for $t \geqslant 0$. 
Lemma 3.1 (Y. Shizuta, I. Vidav). - Let $Y$ be a Banach space and $A$ be a linear operator that generates a strongly continuous semigroup on $Y$ such that $\|\exp (-t A)\| \leqslant$ $M$ for all $t \geqslant 0$. Let $K$ be an A-smoothing operator from $Y$ to $Y$. Then $(A+K)$ generates a strongly continuous semigroup $\exp (-t(A+K))$ and $\sigma(-A-K)$ consists of a finite number of eigenvalues of finite multiplicities in $\{\mathcal{R} e \lambda>\delta\}$ for all $\delta>0$. These eigenvalues can be labeled by

$$
\mathcal{R} e \lambda_{1} \geqslant \mathcal{R} e \lambda_{2} \geqslant \cdots \geqslant \mathcal{R} e \lambda_{N} \geqslant \delta
$$

Furthermore, for every $\Lambda>\mathcal{R} e \lambda_{1}$, there is a constant $C_{\Lambda}$ such that

$$
\|\exp (-t(A+K))\|_{L(Y, Y)} \leqslant C_{\Lambda} \exp (\Lambda t)
$$

Combining these two results, we get in particular that we can always find for bounded $|k|$ an open set $\Omega$ of the form (8), with $\alpha_{o}$ arbitrarily close to the spectral radius $\sigma(k)$.

\section{Ekman layers}

\subsection{Introduction}

Let us study the limit $\varepsilon \rightarrow 0$ of the incompressible Navier-Stokes equations with Coriolis force

$$
\begin{gathered}
\partial_{t} u^{\varepsilon}+\left(u^{\varepsilon} \cdot \nabla\right) u^{\varepsilon}-v \Delta u^{\varepsilon}+\frac{e \times u^{\varepsilon}}{\varepsilon}+\nabla p^{\varepsilon}=0, \\
\operatorname{div} u^{\varepsilon}=0, \\
u^{\varepsilon}=0 \quad \text { on } z=0 \text { and } z=1,
\end{gathered}
$$

in $\Omega=\mathbb{T}_{x, y}^{2} \times[0,1]_{z}$ where $e=(0,0,1)$ denotes a fixed vector, $v>0$ the viscosity, and $\varepsilon$ the Rossby number. Following classical parameter orderings, we assume that $v=\beta \varepsilon$ where $\beta>0$ is a given constant. The limit $\varepsilon \rightarrow 0$ of (40), (41) and (42) has been studied recently in $[9,2]$ and we refer to $[6,14]$ for a physical approach.

In view of Taylor Proudman theorem, the formal limit $u$ of $u^{\varepsilon}$ is a two components two-dimensional flow (independent of $z$ )

$$
u(t, x, y)=\left(\begin{array}{c}
u_{1}(t, x, y) \\
u_{2}(t, x, y) \\
0
\end{array}\right)
$$

which satisfies the two-dimensional Euler equations in $\mathbb{T}_{x, y}^{2}$ with damping term

$$
\begin{gathered}
\partial_{t} u+(u \cdot \nabla) u+\sqrt{2 \beta} u+\nabla p=0, \\
\operatorname{div} u=0 .
\end{gathered}
$$

Since $u$ does not satisfy in general the boundary conditions (42), we have to add boundary layer correctors at $z=0$ and at $z=1$

$$
u_{0}^{B L}\left(t, x, y, z_{r}\right)=-\exp \left(-z_{r}\right)\left(u \cos z_{r}+u^{\perp} \sin z_{r}\right)
$$


where

$$
z_{r}=\frac{z}{\lambda}, \quad \lambda=\sqrt{2 \varepsilon v},
$$

and similarly near $z=1$. It is possible to go further in the construction and to get for every arbitrarily large $N$ an approximate solution $u^{a p p}$ of the form

$$
\begin{aligned}
u^{a p p}(t, x, y, z)= & \sum_{j=0}^{N} \varepsilon^{j} u_{j}^{i n t}(t, x, y)+\sum_{j=0}^{N} \varepsilon^{j} u_{0, j}^{B L}\left(t, x, y, \frac{z}{\lambda}\right) \\
& +\sum_{j=0}^{N} \varepsilon^{j} u_{1, j}^{B L}\left(t, x, y, \frac{1-z}{\lambda}\right)
\end{aligned}
$$

which satisfies (41), (42) and

$$
\partial_{t} u^{a p p}+\left(u^{a p p} \cdot \nabla\right) u^{a p p}-v \Delta u^{a p p}+\frac{e \times u^{a p p}}{\varepsilon}+\nabla p^{a p p}=R^{a p p}
$$

with

$$
\left\|R^{a p p}(t)\right\|_{L^{2}} \leqslant C(t) \varepsilon^{N-1 / 2},
$$

where $C \in L_{\text {loc }}^{\infty}\left(\mathbb{R}^{+}\right)$and $L^{p}(1 \leqslant p \leqslant \infty)$ stands for $L^{p}(\Omega)$. We then get as in [9].

THEOREM 4.1. - Let $u^{\varepsilon}(0)$ be a sequence of $L^{2}$ functions such that

$$
\left\|u^{\varepsilon}(0)-u^{a p p}(0)\right\|_{L^{2}} \leqslant C \varepsilon^{N},
$$

and let $u^{\varepsilon}(t)$ be a corresponding weak solution of (40), (41) and (42) with initial data $u^{\varepsilon}(0)$. Then

$$
\sup _{t \in[0, T]}\left\|u^{\varepsilon}(t)-u^{a p p}(t)\right\|_{L^{2}} \leqslant C_{T} \varepsilon^{N}
$$

for every time $T$ such that

$$
\sup _{0 \leqslant t \leqslant T}\left|u_{0}^{i n t}(t)\right|_{L^{\infty}} \sqrt{\frac{2 \varepsilon}{v}} \leqslant R e_{1},
$$

where $\operatorname{Re}_{1}$ is an universal constant, and $C_{T}$ a nondecreasing function independent of $\varepsilon$.

In [2], the following analytic expression for $R e_{1}$ was given

$$
R e_{1}=\frac{\sqrt{2}}{\int_{0}^{\infty} z(|\cos z|+|\sin z|) \exp (-z) \mathrm{d} z} \sim 1.102 .
$$

In other words, a Reynolds number attached to the boundary layer can be defined as

$$
\operatorname{Re}_{B L}(t, x, y)=\left|u_{0}^{i n t}(t, x, y)\right| \frac{\lambda}{v}
$$


where $\lambda$ denotes the size of the layer. In the present case, $\lambda=\sqrt{2 \varepsilon \nu}$, hence

$$
\operatorname{Re}_{B L}(t, x, y)=\left|u_{0}^{i n t}(t, x, y)\right| \sqrt{\frac{2 \varepsilon}{v}} .
$$

Theorem 4.1 means that as long as the boundary layer Reynolds number $\operatorname{Re}_{B L}(t)=$ $\left|\operatorname{Re}_{B L}(t, \cdot)\right|_{L^{\infty}\left(\mathbb{T}^{2}\right)}$ is small enough, then the Ekman layer is stable and remains laminar, as physically expected. In fact, we proved in [9]

THEOREM 4.2. - Let $u(0) \in H^{4}\left(\mathbb{T}^{2}\right)$ and let $u(t)$ be the corresponding global strong solution of the 2-D Euler equations (43) and (44). Let $u^{\varepsilon}(0)$ be a sequence of initial data bounded in $L^{2}$ and let $u^{\varepsilon}(t)$ be the corresponding sequence of solutions of (40), (41) and (42). If

$$
\left\|u^{\varepsilon}(0)-u(0)\right\|_{L^{2}} \rightarrow 0, \quad \text { then } \sup _{t \in[0, T]}\left\|u^{\varepsilon}(t)-u(t)\right\|_{L^{2}} \rightarrow 0
$$

for every $T$ such that

$$
\sup _{0 \leqslant t \leqslant T} \operatorname{Re}_{B L}(t) \leqslant R e_{1} .
$$

Let us observe that the regularity of solutions of the 2-D Euler equations [20] allows to replace the assumption $u(0) \in H^{4}\left(\mathbb{T}^{2}\right)$ by $u(0) \in L^{2}\left(\mathbb{T}^{2}\right)$ and $\omega(0)=u(0) \in L^{\infty}\left(\mathbb{T}^{2}\right)$.

\subsection{Formalism}

Let us now turn to cases where $R e_{B L}$ is large. First, we put Ekman layers into the formalism developped in the first section. We take $v=\varepsilon$ and consider a vector $e$ colinear to $(0,0,1)$ but not necessarily of unit length. Let

$$
Q\left(v_{1}, v_{2}\right)=P\left(v_{1} \cdot \nabla v_{2}\right), \quad L v_{1}=P e \times v_{1},
$$

and

$$
A u=P\left(\left(u^{a p p} \cdot \nabla\right) u+(u \cdot \nabla) u^{a p p}\right)+\frac{L u}{\varepsilon}-\varepsilon \Delta u .
$$

For $\left(x_{o}, y_{o}\right) \in \mathbb{R}^{2}$ we define

$$
\begin{aligned}
A_{x_{o}, y_{o}}^{r} u= & P_{r}\left(\left(\left(u^{i n t}\left(x_{o}, y_{o}\right)+u_{0}^{B L}\left(x_{o}, y_{o}, z_{r}\right)\right) \cdot \nabla_{r}\right) u+u_{3} \partial_{z_{r}} u_{0}^{B L}\left(x_{o}, y_{o}, z_{r}\right)\right) \\
& +L u-\Delta u
\end{aligned}
$$

namely we freeze the $x$ and $y$ variables in the main boundary layer part of $A$ and rescale it. Eqs. (40), (41) and (42) can be rewritten as (1), linearized equations near $u^{a p p}$ as (4), and $v=u-u^{a p p}$ satisfies (6). By rescaling Eq. (4), we formally come up at leading order with

$$
\partial_{t} w+A_{x_{o}, y_{o}}^{r} w=0 .
$$


More precisely, let $\bar{u}_{r}$ be the rescaled velocity profile (independent of $x_{r}$ and $y_{r}$ ). Note that $\bar{u}_{r}$ is given by

$$
\bar{u}_{r}=-U_{\infty}\left(\begin{array}{c}
\cos \gamma-\exp \left(-z_{r} \sqrt{\frac{\beta}{2}}\right) \cos \left(z_{r} \sqrt{\frac{\beta}{2}}+\gamma\right) \\
-\sin \gamma+\exp \left(-z_{r} \sqrt{\frac{\beta}{2}}\right) \sin \left(z_{r} \sqrt{\frac{\beta}{2}}+\gamma\right) \\
0
\end{array}\right)
$$

where $\gamma$ is an angle. Using the translation invariance of the equation, we may assume that $x_{o}=y_{o}=0$. In this case

$$
A_{x_{o}, y_{o}}^{r} v=A_{0}^{r} v=P_{r}\left(\bar{u}_{r} \cdot \nabla_{r}\right) v+P_{r}\left(v \cdot \nabla_{r}\right) \bar{u}_{r}-\Delta_{r} v+P_{r} e \times v,
$$

$P_{r}$ being the Leray projector on divergence free vector fields.

\subsection{Spectral analysis in $L^{2}$}

Let us first investigate the spectrum of the linearized operator in $L^{2}$. Shizuta and Vidav's Lemma is very useful to get bounds on the spectral radius of $A_{x_{o}, y_{o}}^{r}$. More precisely, let

$$
K_{r} v=P_{r}\left(v \cdot \nabla_{r} \bar{u}_{r}\right),
$$

and

$$
\tilde{A} v=P_{r}\left(-\Delta_{r} v+e \times v+\left(\bar{u}_{r} \cdot \nabla_{r}\right) v\right) .
$$

First we see that $\tilde{A}$ with boundary condition $v=0$ generates a semigroup in $L^{2}$ with $\left\|\exp \left(-t_{r} \tilde{A}\right)\right\|_{L^{2} \rightarrow L^{2}} \leqslant 1$. This semigroup is compact for $t_{r}>0$ and $K$ is a bounded operator from $L^{2} \rightarrow L^{2}$. We can apply Lemma 3.1 to obtain that for any $\alpha>0$ there exists a finite number of eigenvalues with real part greater than $\alpha$.

For given $k_{r}$ and $\gamma$, let $\sigma\left(k_{r}, \gamma\right)$ be the supremum (which is finite) of the imaginary part of the spectrum, and let $\sigma=\sup _{k_{r}, \gamma} \sigma\left(k_{r}, \gamma\right)$. Numerical experiments have been achieved by Lilly in [14] (see also [6,1]): $\sigma$ can be computed and is positive if and only if $R e=U_{\infty} \sqrt{2 \varepsilon / v}$ is greater than a critical Reynolds number $R e_{c} \sim 54.2$ (see [1]). Therefore Ekman layers are linearly unstable whenever $R e=U_{\infty} \sqrt{2 \varepsilon / v}>R e_{c}$ with $R e_{c} \sim 54.2$.

Moreover, $\sigma\left(k_{r}, \gamma\right)$ is smooth near its maximum. Numerically, this maximum is nondegenerate (which is a necessary assumption to use Theorem 2.3).

\subsection{Analysis of the resolvent}

Let us try to solve the resolvent equation in $V_{k}$

$$
\left(A_{0}^{r}-|k| c I d\right)\left(v \exp \left(i k \cdot\left(x_{r}, y_{r}\right)\right)\right)=w \exp \left(i k \cdot\left(x_{r}, y_{r}\right)\right)
$$

where $w \in V_{k}$ is given and $c \in \mathbb{C}$. Note the presence of $|k|$ in factor of $c$ which is traditional. Now make an orthonormal change of variables $\left(x_{r}, y_{r}\right) \rightarrow\left(x_{r}^{\prime}, y_{r}^{\prime}\right)$ such that 
$y_{r}^{\prime}=\left(x_{r}, y_{r}\right) \cdot k /|k|$. In the new variables, $\exp \left(i k \cdot\left(x_{r}, y_{r}\right)\right) \hat{v}\left(k, z_{r}\right)=\exp \left(i K y_{r}^{\prime}\right) \hat{v}\left(k, z_{r}\right)$, where $K:=|k|$. As this vector field is independent of $x_{r}^{\prime}$, we can introduce a stream function $\Psi$ and a function $U$ such that

$$
\exp \left(i k \cdot\left(x_{r}, y_{r}\right)\right) \hat{v}\left(k, z_{r}\right)=\exp \left(i K y_{r}^{\prime}\right)\left(\begin{array}{c}
U\left(z_{r}\right) \\
\Psi^{\prime}\left(z_{r}\right) \\
-i K \Psi\left(z_{r}\right)
\end{array}\right) .
$$

System (55) then reduces to the following four by four system on the two functions $(U, \Psi)$

$$
\begin{aligned}
& \quad-\frac{\mathrm{d}^{2} U}{\mathrm{~d} z_{r}^{2}}+K^{2} U-2 \frac{\mathrm{d} \Psi}{\mathrm{d} z_{r}}+i K \operatorname{Re}\left(\left(v_{l}+i c\right) U-\Psi \frac{\mathrm{d} u_{l}}{\mathrm{~d} z_{r}}\right)=w_{1}, \\
& \left(\frac{\mathrm{d}^{2}}{\mathrm{~d} z_{r}^{2}}-K^{2}\right)^{2} \Psi-i K \operatorname{Re}\left(\left(v_{l}+i c\right)\left(\frac{\mathrm{d}^{2}}{\mathrm{~d} z_{r}^{2}}-K^{2}\right) \Psi-\Psi \frac{\mathrm{d}^{2} v_{l}}{\mathrm{~d} z_{r}^{2}}\right)-2 \frac{\mathrm{d} U}{\mathrm{~d} z_{r}} \\
& =i K w_{3}-\frac{\mathrm{d} w_{2}}{\mathrm{~d} z_{r}}
\end{aligned}
$$

where

$$
u_{l}=\cos \tilde{\gamma}-\exp \left(-z_{r} \sqrt{\frac{\beta}{2}}\right) \cos \left(\tilde{\gamma}+z_{r} \sqrt{\frac{\beta}{2}}\right)
$$

and

$$
v_{l}=-\sin \tilde{\gamma}+\exp \left(-z_{r} \sqrt{\frac{\beta}{2}}\right) \sin \left(\tilde{\gamma}+z_{r} \sqrt{\frac{\beta}{2}}\right),
$$

$\tilde{\gamma}$ being an angle between the direction of the flow outside the boundary layer and the direction of $k$, with boundary conditions

$$
U(0)=0, \quad \Psi(0)=\frac{\mathrm{d} \Psi}{\mathrm{d} z_{r}}(0)=0
$$

on $z_{r}=0$ and

$$
\frac{\mathrm{d} U}{\mathrm{~d} z_{r}}=\frac{\mathrm{d}^{2} \Psi}{\mathrm{d} z_{r}^{2}}=0
$$

at infinity.

We already know that if $\mathcal{I} m c>0$ system (56) and (57) can be solved in $L^{2}$ except for a finite number of values of $c$ (given by Lemma 3.1), which are eigenvalues with finite multiplicities. Apart from these eigenvalues, the solution to (56) and (57) is unique, and is easily seen to belong to $H^{s}$. Moreover, the solution is continuous and infinitely many times differentiable with respect to $k$, as soon as we are away from the spectrum. Since $A_{0}^{r}$ is a sectorial operator, we can choose a contour of the form $\partial \Omega$ as soon as $\alpha_{o}$ is greater than $\sigma$. Hence assumption (A4) holds true. 


\subsection{Instability results}

Let us now restate Theorem 2.3 in the case of Ekman layers:

THEOREM 4.3 (in rescaled variables). - Let $\bar{u}_{r}$ be the velocity profile defined by (51). If $0<\sigma<+\infty$ and is nondegenerate, then $\bar{u}_{r}$ is linearly unstable. It is also nonlinearly unstable in the following sense: for every arbitrarily large $s$, and every arbitrarily small $\delta>0$, there exists a solution $u_{r}^{\delta}(t)$ of the rescaled version of (40), (41) and (42) such that

$$
\left\|u_{r}^{\delta}(0)-\bar{u}_{r}\right\|_{H^{s}} \leqslant \delta
$$

and

$$
\left\|u_{r}^{\delta}\left(T_{r}^{\delta}\right)-\bar{u}_{r}\right\|_{L^{\infty}} \geqslant \alpha>0, \quad\left\|u_{r}^{\delta}\left(T_{r}^{\delta}\right)-\bar{u}_{r}\right\|_{L^{2}} \geqslant \alpha>0
$$

for some positive time $T_{r}^{\delta}$, where $\alpha>0$ is independent of $\delta$. Moreover we can choose $u_{r}^{\delta}$ and $T_{r}^{\delta}$ such that $T_{r}^{\delta} \sim C_{s} \log \delta^{-1}$.

Note that numerical computations show that the assumptions are satisfied as soon as the Reynolds number is larger than $R e_{c} \sim 54.2$. Therefore, for sufficiently high Reynolds numbers, the Ekman layer is unstable and undergoes a transition from laminar layer to "turbulent" layer. The main open problem is to know whether this turbulent layer will separate from the wall and enter into the fluid or if it dissipates more than the laminar layer, which in both case would prevent a $L^{2}$ convergence to (43) and (44) in the interior, or if it will remain confined near the wall. Physical experiments [6] seem to indicate that the layer separates from the wall and that waves propagate. However, these experiments are carried out at relatively "large" layers and relatively large parameter values of $v$.

This theorem can be refined to get

THEOREM 4.4 (in original variables). - Let us assume that $0<\sigma<+\infty$ and is nondegenerate for $R e>R e_{c}$. Let $u^{a p p}$ be an approximate solution defined as in (2). Let us assume that

$$
\left\|u^{a p p}(0)\right\|_{L^{\infty}} \sqrt{\frac{2 \varepsilon}{v}}>\operatorname{Re}_{c} .
$$

Then $u^{a p p}$ is nonlinearly unstable in the following sense: for every arbitrarily large $s$, and every arbitrarily small $\varepsilon>0$, there exist two solutions $u_{1}^{\varepsilon}$ and $u_{2}^{\varepsilon}$ of (40), (41) and (42) such that

$$
\left\|u_{1}^{\varepsilon}(0)-u^{a p p}(0)\right\|_{H^{s}}+\left\|u_{2}^{\varepsilon}(0)-u^{a p p}(0)\right\|_{H^{s}} \leqslant C \varepsilon^{N}
$$

and

$$
\left\|u_{1}^{\varepsilon}\left(T^{\varepsilon}\right)-u_{2}^{\varepsilon}\left(T^{\varepsilon}\right)\right\|_{L^{\infty}} \geqslant \alpha>0
$$

for some positive time $T^{\varepsilon}$, where $\alpha>0$ is independent of $\varepsilon$, and $T^{\varepsilon}$ goes to 0 as $\varepsilon$ goes to 0 .

Proof of the theorems. - Theorems 4.3 and 4.4 are just restatements of Theorem 2.3. Using the divergence free condition and the explicit form of $Q,(\mathrm{~A} 1)$ and (A3) are 
straightforward, and (A2) is the linear instability which is an assumption. Finally, (A4) has been proven in Section 4.4.

\section{Application to Hartman-Ekman layers}

In geophysical situations, we are interested in the following MHD equations on the fluid velocity $u$ and the magnetic field $b$, in a domain $\Omega=\mathbb{R}^{2} \times[0,1]$, rapidly rotating around $e_{3}$, and within a strong external fixed magnetic field with direction $e_{3}$,

$$
\begin{gathered}
\partial_{t} u+(u \cdot \nabla) u+\frac{\nabla p}{\varepsilon}-\frac{E}{\varepsilon} \Delta u+\frac{e_{3} \times u}{\varepsilon}=\frac{\Lambda}{\varepsilon} \operatorname{curl} b \times e_{3}+\frac{\Lambda \theta}{\varepsilon} \operatorname{curl} b \times b, \\
\partial_{t} b+(u \cdot \nabla) b=b \cdot \nabla u+\frac{\operatorname{curl}\left(u \times e_{3}\right)}{\theta}+\frac{\Delta b}{\theta}, \quad \operatorname{div} b=0, \quad \operatorname{div} u=0,
\end{gathered}
$$

and in $\Omega^{c}$ we consider the Maxwell type equations

$$
\operatorname{curl} b=0, \quad \operatorname{curl} E=-\theta \partial_{t} b, \quad \operatorname{div} E=0, \quad \operatorname{div} b=0 .
$$

The boundary conditions are

$$
u=0 \text { and } E \times b \text { is continuous, on } z=0 \text { and } z=1 .
$$

Note in particular that on the fluid's side, we have curl $b=E$ on $z=0$ and $z=1$. We consider the following physically relevant orderings for $E, \Lambda, \theta, \varepsilon$

$$
\varepsilon \rightarrow 0, \quad \Lambda=\mathrm{O}(1), \quad \theta \rightarrow 0, \quad E \sim \varepsilon^{2} .
$$

The Reynolds number in this case reads as

$$
\operatorname{Re}=\frac{\left\|u_{0}^{i n t}\right\|_{L^{\infty}\left(\mathbb{R}^{2}\right)} \varepsilon}{\sqrt{E}}
$$

and in [2] we proved the stability of the boundary layers for small Reynolds. In [3] we computed the critical Reynolds number. A Theorem similar to 4.4 holds true and can be proved with the same ideas. Pure Hartmann layers (external strong fixed magnetic field without rotation) can also be treated. More difficult situations where the rotation vector of the domain is not perpendicular to the boundary or where the external magnetic field is not directed along $e_{3}$ can also be handled, and are of geophysical interest [3].

We will not detail here this application nor state a precise result since it completly follows the lines of the Ekman case.

\section{Acknowledgements}

The authors would like to thank Y. Guo for interesting discussions on nearby subjects. 


\section{REFERENCES}

[1] B. Desjardins, E. Grenier, Reynolds.m a package to compute critical Reynolds numbers, 1998, http://www.dmi.ens.fr/equipes/edp/Reynolds/reynolds.html.

[2] E. Dormy, B. Desjardins, E. Grenier, Stability of mixed Ekman-Hartmann boundary layers, Nonlinearity 12 (2) (1999) 181-199.

[3] E. Dormy, B. Desjardins, E. Grenier, Instability of Ekman-Hartmann boundary layers, with application to the fluid flow near the core-mantle boundary, Physics of the Earth and Planetary Interiors 123 (2001) 15-26.

[4] S. Friedlander, W. Strauss, M. Vishik, Nonlinear instability in an ideal fluid, Ann. Inst. H. Poincaré Anal. Non Linéaire 14 (1997) 187-209.

[5] M. Gisclon, D. Serre, Study of boundary conditions for a strictly hyperbolic system via parabolic approximation, C. R. Acad. Sci. Paris Ser. I Math. 319 (4) (1994) 377-382.

[6] H.P. Greenspan, The Theory of Rotating Fluids, in: Cambridge Monographs on Mechanics and Applied Mathematics, 1969.

[7] E. Grenier, On the nonlinear instability of Euler and Prandtl equations, Comm. Pure Appl. Math. 53 (2000) 1067-1091.

[8] E. Grenier, O. Guès, Boundary layers for viscous perturbations of noncharacteristic quasilinear hyperbolic problems, J. Differential Equations 143 (1) (1998) 110-146.

[9] E. Grenier, N. Masmoudi, Ekman layers of rotating fluids, the case of well prepared initial data, Comm. Partial Differential Equations 22 (1997) 953-975.

[10] Y. Guo, W. Strauss, Instability of periodic BGK equilibria, Comm. Pure Appl. Math. 48 (1995) 861-894.

[11] Y. Guo, W. Strauss, Nonlinear instability of double-humped equilibria, Ann. Inst. H. Poincaré Anal. Non Linéaire 12 (1995) 339-352.

[12] D. Henry, Geometric Theory of Semilinear Parabolic Equations, in: Lecture Notes in Mathematics, Vol. 840, Springer, Berlin, 1981.

[13] G. Iooss, H.B. Nielsen, H. True, Bifurcation of the stationary Ekman flow into a stable periodic flow, Arch. Rational Mech. Anal. 68 (3) (1978) 227-256.

[14] D.K. Lilly, On the instability of the Ekman boundary layer, J. Atmos. Sci. 23 (1966) 481494.

[15] A. Majda, Compressible Fluid Flows Systems of Conservation Laws in Several Variables, in: Appl. Math. Sci., Vol. 53, Springer, Berlin, 1984.

[16] D. Serre, $L^{1}$-stability of travelling waves in scalar conservation laws, Exp. No. VIII, 13 pp., Semin. Equ. Dériv. Partielles, Ecole Polytech., Palaiseau, 1999.

[17] D. Serre, Systèmes de lois de conservations, I et II, Diderot Editeur, Paris, 1996.

[18] Y. Shizuta, On the classical solutions of the Boltzmann equation, Comm. Pure Appl. Math. 36 (1983) 705-754.

[19] I. Vidav, Spectra of perturbed semigroups with applications to transport theory, J. Math. Anal. Appl. 30 (1970) 264-279.

[20] V.I. Yudovitch, Non-stationary flow of a perfect non-viscous fluid, Zh. Vych. Math. 3 (1963) 1032-1066. 